\title{
Is Angiostrongylosis a Realistic Threat for Domestic Cats?
}

\author{
Angela Di Cesare ${ }^{1}$, Simone Morelli ${ }^{1}$, Mariasole Colombo ${ }^{1}$, Giulia Simonato ${ }^{2}$, \\ Fabrizia Veronesi ${ }^{3}$, Federica Marcer ${ }^{2}$, Anastasia Diakou ${ }^{4}$, Roberto D'Angelosante ${ }^{1}$, \\ Nikola Pantchev ${ }^{5}$, Evanthia Psaralexi ${ }^{4}$ and Donato Traversa ${ }^{1 *}$

\begin{abstract}
${ }^{1}$ Faculty of Veterinary Medicine, University of Teramo, Teramo, Italy, ${ }^{2}$ Department of Animal Medicine, Production and Health, University of Padua, Padua, Italy, ${ }^{3}$ Department of Veterinary Medicine, University of Perugia, Perugia, Italy, ${ }^{4}$ Faculty of Veterinary Medicine, Aristotle University of Thessaloniki, Thessaloniki, Greece, ${ }^{5}$ IDEXX Laboratories, Ludwigsburg, Germany
\end{abstract}

Three species of Angiostrongylus have been found in felids thus far, i.e., Angiostrongylus chabaudi, Angiostrongylus felineus and Angiostrongylus vasorum. Angiostrongylus chabaudi lives in the right heart and pulmonary arteries of the definitive natural host, the European wildcat (Felis silvestris), and non-patent infections have been reported in domestic cats (Felis catus). Angiostrongylus felineus, described in the Puma yaguarondi (Herpailurus yagouaroundi), has never been reported in domestic felids, while recently a non-patent infection by $A$. vasorum was unequivocally described in a $F$. catus.

\section{OPEN ACCESS}

Edited by:

Olivier Andre Sparagano, City University of Hong Kong,

Hong Kong

Reviewed by:

Andrei Daniel Mihalca, University of Agricultural Sciences and Veterinary Medicine of

Cluj-Napoca, Romania Abdul Jabbar,

The University of Melbourne, Australia

*Correspondence: Donato Traversa dtraversa@unite.it

Specialty section: This article was submitted to Parasitology, a section of the journal Frontiers in Veterinary Science

Received: 12 February 2020 Accepted: 25 March 2020

Published: 15 April 2020

Citation:

Di Cesare A, Morelli S, Colombo M, Simonato G, Veronesi $F$, Marcer $F$,

Diakou A, D'Angelosante R,

Pantchev N, Psaralexi E and Traversa D (2020) Is Angiostrongylosis a Realistic Threat for Domestic Cats?

Front. Vet. Sci. 7:195 doi: 10.3389/fvets.2020.00195 Nonetheless, epizootiological and clinical relevance of angiostrongylosis in domestic cats are practically unknown. This study investigated whether cases of angiostrongylosis may be missed in cats living in areas enzootic for Angiostrongy/us spp. and other metastrongyloids. Overall, 100 cats that were either positive (n.50) or negative (n.50) for metastrongyloid larvae at the Baermann's test, were examined for Angiostrongylus spp. with DNA-based methods and with the serological test Angio Detect ${ }^{\mathrm{TM}}$ for circulating antigen. The PCR analysis confirmed the copromicroscopy results, where 25 cats scored positive for Aelurostrongylus abstrusus, 16 for Troglostrongylus brevior and 9 for both, while no cats were positive for Angiostrongy/us-like larvae, including $A$. chabaudi. None of the 100 sera samples scored positive at the Angio Detect ${ }^{\mathrm{TM}}$ test. These data suggest that currently feline angiostrongylosis is a minor parasitosis for domestic cats. Nevertheless, it cannot be excluded that the epizootiological drivers which have favored the spillover of A. vasorum and T. brevior from wildlife to dogs and cats, could promote the emergence of feline angiostrongylosis, with an unpredictable health impact.

Keywords: Angiostrongylus chabaudi, wildcat, cat, angiostrongylosis, angio detect ${ }^{\mathrm{TM}}$

\section{INTRODUCTION}

Canine and feline cardio-pulmonary nematodes are emerging throughout Europe due to different factors $(1,2)$. A role in the spreading of some extra-intestinal parasites can be played by bridging infections between wildlife and domestic hosts $(3,4)$. This has recently led to the establishment in domestic animals of parasites which were previously considered to be affiliated only to their definitive wild hosts. As key examples, among these "new" parasites, Angiostrongylus vasorum causing canine angiostrongylosis, Troglostrongylus brevior causing feline troglostrongylosis and Oslerus rostratus causing feline oslerosis were previously considered typical of red foxes (Vulpes vulpes), European wildcats (Felis silvestris) and the lynx (Lynx spp.), respectively. These nematodes have 
now a major impact on animal health, causing potentially fatal diseases in domestic canine and feline populations (4-6).

While angiostrongylosis in dogs has become a well-known disease in Europe, our knowledge on the infections caused by Angiostrongylus spp. in felines is very poor. Angiostrongylus chabaudi is a metastrongyloid nematode that was first described in wildcats from Central Italy in 1957 (7), and that has not been reported again until few years ago, when it was found in a domestic cat (Felis catus) from Sardinia (Italy) as a non-patent infection (8). Then, this parasite has been documented in another non-patently infected domestic cat from Central Italy (9) and in wildcats from Germany (10), Italy (11), Greece $(12,13)$, Romania (14), Bulgaria (15), Bosnia and Herzegovina (16). Recently, the European wildcat has been shown to be the definitive host of $A$. chabaudi, with a demonstration of patent infections and presence of first stage larvae (L1) in the feces for the first time (12). The clinical importance of angiostrongylosis caused by A. chabaudi in domestic cats is virtually unknown, although pathological lesions (e.g., granulomatous pneumonia, parenchymal hemorrhagies, alveolar emphysema, subendothelial proliferation and oedema, thrombosis and hyperplasia/hypertrophy of the pulmonary arteries) have been reported in both wild and domestic cats (9, 11, 12, 16). With regard to other Angiostrongylus species, the ability of A. vasorum to infect felid hosts was shown under experimental conditions $(17,18)$, and recently, a case of natural infection has been described in a domestic cat from Switzerland (19). However, to date patent infections caused by A. vasorum in $F$. catus have never been described neither in experimental nor in natural conditions. Few years ago, Angiostrongylus felineus has been described for the first time in the Puma yaguarondi (Herpailurus yagouaroundi) from Brazil (20), but at present no cases of infection have been documented in domestic cats.

The diagnosis of feline cardio-respiratory parasitic infections is currently based on the morphometric and morphologic identification of L1 shed in faces using the Baermann test (21). A key limitation in diagnosing possible angiostrongylosis in domestic cats is due to the fact that at the moment there is no any demonstrations of patent infections. This absence of data could have been caused by missed diagnosis due to a certain similarity of microscopic features of Angiostrongylus with those of other metastrongyloids more commonly detected in fecal samples of cats (i.e., Aelurostrongylus abstrusus and T. brevior) (12).

DNA-based assays are powerful to identify metastrongyloid L1s in the feces of cats (22), thus would have the potential to reveal missed diagnosis at the microscopic analysis. The rapid serological test Angio Detect ${ }^{\mathrm{TM}}$ (IDEXX Laboratories Inc.) is able to detect circulating antigens produced by $A$. vasorum infecting dogs (23). Interestingly, this test has recently proved useful for the detection of other Angiostrongylus species, i.e., A. chabaudi in European wildcats and Angiostrongylus daskalovi in badgers (24). Being able to detect Angiostrongylus spp. circulating antigens, this test could also have a potential to diagnose feline angiostrongylosis.

The present study has aimed at investigating for the first time whether angiostrongylosis is a potentially missed, neglected or underestimated disease in domestic cats by using DNA-based and serological tests.

\section{MATERIALS AND METHODS}

One-hundred domestic cats from regions of Italy and Greece enzootic for Angiostrongylus spp. and major feline cardiorespiratory nematodes $(2,9,11-13)$ were examined for the presence of metastrongyloid larvae. Animals were selected as a convenient dataset, as they were referred for routine clinical examinations and/or presence of compatible clinical signs. All animals were not sampled purposely for the present study and were not subjected to unnecessary suffering. Only the surplus material deriving from blood samplings expressly requested by the owner and/or indicated by the examining veterinarian for other diagnostic purposes was used in this study. Healthy animals were sampled providing that they were from enzootic areas and had a free-roaming lifestyle. From one to three consecutive fecal samples were examined for each cat by a Baermann's examination (2). Cats were divided in two groups, i.e., cats that scored either positive (G1) or negative (G2) for metastrongyloid larvae at the fecal test. All larvae collected at the Baermann test were examined using morphological and morphometric keys $(12,21)$. All Baermann's sediments were subjected to a multiplex-PCR as previously described (22) that is able to simultaneously $\operatorname{detect}$ A. chabaudi, Ae. abstrusus, and T. brevior. An individual blood sample was obtained for each cat with the owner's consent. Samples were collected for veterinary hematological examinations (e.g., routine or other diagnostic tests). Samples were subjected to centrifugation to obtain the serum. All sera samples were examined using the Angio Detect ${ }^{\mathrm{TM}}$ test (IDEXX Laboratories, Westbrook, Maine, USA), according to the manufacturer's instructions. More details are reported in Schnyder et al. (23).

\section{RESULTS AND DISCUSSION}

Out of the 50 Baermann-positive cats (G1), 25 were microscopically positive for Ae. abstrusus, 16 for $T$. brevior and nine were positive for both nematodes. None of the samples scored positive for Angiostrongylus-like larvae nor for $O$. rostratus (the latter was not surprising as $O$. rostratus larvae usually do not migrate in water). PCR results confirmed the microscopic identification of the L1s retrieved in the Baermann sediment and there were no PCR-positive for samples negative at the copromicroscopy. All sera samples scored negative for circulating Angiostrongylus antigen at the Angio Detect ${ }^{\mathrm{TM}}$ (Table 1).

TABLE 1 | Results of the Baermann examination confirmed by molecular analysis ${ }^{*}$ ) and of the serological Angio Detect ${ }^{\mathrm{TM}}$ test performed in the present study.

\begin{tabular}{|c|c|c|c|}
\hline & $\begin{array}{l}\text { Italy } \mathrm{n} / \text { tot } \\
\text { (\%) }\end{array}$ & $\begin{array}{c}\text { Greece } n / \text { tot } \\
(\%)\end{array}$ & $\begin{array}{c}\text { Total } n / \text { tot } \\
(\%)\end{array}$ \\
\hline Aelurostrongylus abstrusus* & 23/64 (35.9) & 2/36 (5.5) & $25 / 100(25)$ \\
\hline Troglostrongylus brevior & $15 / 64(23.4)$ & $1 / 36(2.7)$ & $16 / 100(16)$ \\
\hline $\begin{array}{l}\text { Aelurostrongylus abstrusus } \\
+ \text { Troglostrongylus brevior }\end{array}$ & 8/64 (12.5) & $1 / 36(2.7)$ & 9/100 (9) \\
\hline Angiostrongylus chabaudi* & 0/64 (0) & 0/36 (0) & 0/100 (0) \\
\hline Angio Detect ${ }^{\mathrm{TM}}$ & 0/64 (0) & 0/64 (0) & 0/100 (0) \\
\hline
\end{tabular}


Although preliminary, this study suggests that cats living in areas where Angiostrongylus is present even with high infection rates $(2,11-13)$, are realistically at a null or minor risk of acquiring angiostrongylosis. The absence of $A$. chabaudi L1s in potentially infected cats could be due to the inability of this metastrongyloid to reach the adult stage in domestic hosts $(8,9)$. To date, indeed, only two cases of $A$. chabaudi infection have been described in domestic cats, where necropsy examinations showed immature nematodes in the pulmonary arteries without evidence of $\mathrm{L} 1$ in feces $(8,9)$. In a recent survey, the Angio Detect ${ }^{\mathrm{TM}}$ test showed a $97.1 \%$ sensitivity and a $98.9 \%$ specificity in detecting $A$. vasorum infection in dogs (25) and another study carried out on wildcats and badgers positive for A. chabaudi and A. daskalovi, respectively, showed $100 \%$ correlation between necropsy results, confirmed by molecular assays, and serologic positivity to the Angio Detect ${ }^{\mathrm{TM}}$ test (24). The sero-negativity of G1 cats suggests a lack of cross-reactivity between the different lungworm species infecting cats. In fact, it seems that the Angio Detect ${ }^{\mathrm{TM}}$ test can crossreact exclusively between species of the genus Angiostrongylus (24). This might be also explained by their colonization of blood vessels and thus the direct availability of circulating antigen in blood samples, as it is the case with the heartworm, Dirofilaria immitis. It can be assumed that this rapid assay has an elevated sensitivity, although not $100 \%$ (25), either in wildcats or in domestic cats, because its reliability does not seem to be influenced by the tested species, having been used successfully in non-canine hosts (24). Furthermore, the Angio Detect $^{\mathrm{TM}}$ is able to detect the infection also in absence of Angiostrongylus spp. L1 in feces, as long as the parasites have reached adulthood (25). Therefore, it is unlikely that the cats of the present study were false negative, having been tested with three different diagnostic methods and the serologic negativity is most probably due to a real absence of $A$. chabaudi, $A$. vasorum, or other Angiostrongylus species. In fact, cats of G1, shedding larvae, would have scored positive at least at one of the tests performed in the present study. This is supported by the fact that all cats of both G1 and G2 scored negative for Angiostrongylus spp., other than the Angio Detect ${ }^{\mathrm{TM}}$ test, also at the molecular analysis for A. chabaudi, which seems to be the major Angiostrongylus infecting felines. Nonetheless, it should be considered that the antigens detectable with this rapid assay are produced by adult Angiostrongylus worms $(23,26)$ and that $A$. chabaudi and $A$. vasorum are most probably unable to reach adulthood producing detectable antigens in domestic cats $(8,9,19)$. Indeed, a nematode found in a pulmonary artery of a domestic cat, whose section size and content were suggestive of an adult stage, was identified as A. vasorum at PCR analysis despite the cat scored negative at the Angio Detect $^{\mathrm{TM}}$ test (19). Such negativity could be due to different reasons, including a $<100 \%$ sensitivity of the test (25) or to the fact that the first antigens are detectable 9 weeks postinfection (23). As development of A. vasorum to adult may occur as early as 28 days post-infection (27) false negative results might occur with this test even in presence of adult nematodes. The herein examined cats could have scored as false negatives only in a similar scenario, where A. vasorum infection confirmation could be possible only with necropsy and molecular analysis on the nematodes retrieved in the arteries. Although this possibility cannot be ultimately excluded, these results indicate that angiostrongylosis, at present, is still an infection of minor importance for domestic cats. However, possible bridging infections by Angiostrongylus in future, especially A. chabaudi, between wildcats and domestic cats cannot be ruled out. In fact, different factors are influencing domestic and wild fauna interactions, e.g., urbanization and reduction of wildlife habitats (2). Thus, spill-over events similar to those that have likely occurred for A. vasorum in dogs and T. brevior in cats (4, 6) may indicate that similar eco-epizootiological modifications could have the potential to influence biology and epizootiology of related parasites belonging to the genus Angiostrongylus. A possible spreading of feline angiostrongylosis could have a severe clinical relevance in domestic cats, as happened for canine angiostrongylosis. Angiostrongylosis by A. vasorum in foxes, i.e., the natural reservoir, appears to be milder that in dogs, with a moderate impact on the general health status, and with pathological lesions suggestive of a mainly chronic course (2830 ), while canine angiostrongylosis is a well-known disease with a possible fatal outcome (6).

In conclusion, domestic cats seem to be at low risk of angiostrongylosis, even in areas where wildcats and domestic cats live in sympatry and where different species of Angiostrongylus occur.

It is interesting to note also that the worldwide spread land snail Cornu aspersum may act as intermediate host of A. chabaudi, A. vasorum, and Ae. abstrusus (31-33). Despite A. vasorum and Ae. abstrusus may be present in the same geographic areas, possibly developing in C. aspersum, this is not the case of $A$. chabaudi. This could be due to the very low presence of this nematode in its reservoirs, that is also reflected by a low presence of infective stages in mollusks and paratenic hosts.

As future outbreaks of feline angiostrongylosis with an unforeseeable impact on feline health should be taken into account, constant epizootiological surveillance appears to be crucial. The use of Angio Detect ${ }^{\mathrm{TM}}$ is promising for its use in feline medicine under both clinical and epizootiological standpoints although the diagnostic efficiency of this test in cats that have non-patent infections remains to be understood. As the identification of Angiostrongylus L1 in cats can be impaired by the similarity in morphologic and morphometric features with other metastrongyloids that can be usually found at the Baermann's examination $(12,21)$, the use of combined diagnostic approaches including copromicroscopy, DNA-based assays and rapid serological kits may minimize the chances of false negative results and is herein encouraged in cats that could be at risk of infection by Angiostrongylus.

\section{DATA AVAILABILITY STATEMENT}

The datasets generated for this study are available on request to the corresponding author. 


\section{ETHICS STATEMENT}

Ethical approval for this study was not required according to national/local legislation because no cats were sampled or subjected to unnecessary suffering. The animals included were not bled for this study as only the surplus material deriving from blood samplings expressly requested by the owner and/or indicated by the examining veterinarian for other diagnostic purposes was used in this study.

\section{AUTHOR CONTRIBUTIONS}

ADC, SM, and DT participated in study activities and in drafting and revising the manuscript. MC, GS, FV, FM, AD, RD'A, and

\section{REFERENCES}

1. Traversa D, Di Cesare A. Cardio-pulmonary parasitic nematodes affecting cats in Europe: unraveling the past, depicting the present, and predicting the future. Front Vet Sci. (2014) 1:11. doi: 10.3389/fvets.2014.00011

2. Traversa D, Morelli S, Cassini R, Crisi PE, Russi I, Grillotti E, et al. Occurrence of canine and feline extra-intestinal nematodes in key endemic regions of Italy. Acta Trop. (2019) 193:227-35. doi: 10.1016/j.actatropica.2019.03.009

3. Eleni C, De Liberato C, Azam D, Morgan ER, Traversa D. Angiostrongylus vasorum in wolves in Italy. Int J Parasitol Parasites Wildl. (2013) 3:12-14. doi: 10.1016/j.ijppaw.2013.10.003

4. Crisi PE, Di Cesare A, Boari A. Feline troglostrongylosis: current epizootiology, clinical features, and therapeutic options. Front Vet Sci. (2018) 5:126. doi: 10.3389/fvets.2018.00126

5. Traversa D, Di Cesare A. Feline lungworms: what a dilemma. Trends Parasitol. (2013) 29:423-30. doi: 10.1016/j.pt.2013.07.004

6. Elsheikha HM, Holmes SA, Wright I, Morgan ER, Lacher DW. Recent advances in the epidemiology, clinical and diagnostic features, and control of canine cardio-pulmonary angiostrongylosis. Vet Res. (2014) 45:92. doi: 10.1186/s13567-014-0092-9

7. Biocca E. Angiostrongylus chabaudi n. sp. parassita del cuore e dei vasi polmonari del gatto selvatico (Felis silvestris). $R$ Accad Naz Lincei. (1957) 22:526-32.

8. Varcasia A, Tamponi C, Brianti E, Cabras PA, Boi R, Pipia AP, et al. Angiostrongylus chabaudi Biocca, 1957: a new parasite for domestic cats? Parasit Vectors. (2014) 7:588. doi: 10.1186/s13071-014-0588-1

9. Traversa D, Lepri E, Veronesi F, Paoletti B, Simonato G, Diaferia M, et al. Metastrongyloid infection by Aelurostrongylus abstrusus, Troglostrongylus brevior and Angiostrongylus chabaudi in a domestic cat. Int J Parasitol. (2015) 45:685-90. doi: 10.1016/j.ijpara.2015.05.005

10. Steeb S, Hirzmann J, Eskens U, Volmer K, Bauer C. Lungenwurm-infektionen bei der europäischen wildkatze. Kompakt Vet. (2014) 3.

11. Veronesi F, Traversa D, Lepri E, Morganti G, Vercillo F, Grelli D, et al. Occurrence of lungworms in european wildcats (Felis silvestris silvestris) of central Italy. J Wild Dis. (2016) 52:270-8. doi: 10.7589/2015-07-187

12. Diakou A, Psalla D, Migli D, Di Cesare A, Youlatos D, Marcer F, et al. First evidence of the European wildcat (Felis silvestris silvestris) as definitive host of Angiostrongylus chabaudi. Parasitol Res. (2016) 115:1235-44. doi: 10.1007/s00436-015-4860-x

13. Diakou A, Dimzas D, Astaras C, Savvas I, Di Cesare A, Morelli S, et al. Clinical investigations and treatment outcome in a European wildcat (Felis silvestris silvestris) infected by cardio-pulmonary nematodes. Vet Parasitol Reg Stud Rep. (2019) 19:100357. doi: 10.1016/j.vprsr.2019.100357

14. Gherman CM, Ionica AM, D'Amico G, Otranto D, Mihalca AD. Angiostrongylus chabaudi (Biocca, 1957) in wildcat (Felis silvestris silvestris, S) from Romania. Parasitol Res. (2016) 115:2511-7. doi: 10.1007/s00436-016-5032-3
EP participated in the field and laboratory work. NP participated in the study design and in interpreting the serological results. All authors have participated in critically revising the manuscript.

\section{FUNDING}

IDEXX has kindly provided the Angio Detect ${ }^{\mathrm{TM}}$ kits used in the present study.

\section{ACKNOWLEDGMENTS}

The authors are grateful to Dr. Barbara Paoletti and Dr. Raffaella Iorio for their support to the laboratory activities of the present study.

15. Giannelli A, Kirkova Z, Abramo F, Latrofa MS, Campbell B, Zizzo N, et al. Angiostrongylus chabaudi in felids: new findings and a review of the literature. Vet Parasitol. (2016) 228:188-92. doi: 10.1016/j.vetpar.2016.09.007

16. Stevanović O, Diakou A, Morelli S, Paraš S, Trbojević I, Nedić D, et al. Severe Verminous Pneumonia Caused by Natural Mixed Infection with Aelurostrongylus abstrusus and Angiostrongylus chabaudi in a European Wildcat from Western Balkan Area. Acta Parasitol. (2019) 64:411-7. doi: 10.2478/s11686-019-00029-9

17. Guilhon J, Cens B. Attempts of transmission of Angiostrongylus vasorum (Baillet 1886) to the cat (in French). C R Acad Hebd Seances Acad Sci D. (1970) 271:936-9.

18. Dias SR, Oliveira EI, Viana MH, Lima WS. Permissivity of the domestic cat (Felis catus) to infection by Angiostrongylus vasorum (Nematoda: Protostrongylidae). Rev Méd Vét. (2008) 159:87-90.

19. Gueldner EK, Schuppisser C, Borel N, Hilbe M, Schnyder M. First case of a natural infection in a domestic cat (Felis catus) with the canid heart worm Angiostrongylus vasorum. Vet Parasitol Reg Stud Rep. (2019) 18:100342. doi: 10.1016/j.vprsr.2019.100342

20. Vieira FM, Muniz-Pereira LC, de Souza Lima S, Neto AH, Guimarães EV, Luque JL. A new metastrongyloidean species (Nematoda) parasitizing pulmonary arteries of Puma (Herpailurus) yagouaroundi (É. Geoffroy, 1803) (Carnivora: Felidae) from Brazil. J Parasitol. (2013) 99:327-31. doi: $10.1645 / G E-3171.1$

21. Traversa D, Di Cesare A. Diagnosis and management of lungworm infections in cats: cornerstones, dilemmas and new avenues. J Fel Med Surg. (2016) 18:7-20. doi: 10.1177/1098612X15623113

22. Di Cesare A, Veronesi F, Frangipane di Regalbono A, Iorio R, Traversa D. Novel molecular assay for simultaneous identification of neglected lungworms and heartworms affecting cats. J Clin Microbiol. (2015) 53:300913. doi: 10.1128/JCM.00901-15

23. Schnyder M, Stebler K, Naucke TJ, Lorentz S, Deplazes P. Evaluation of a rapid device for serological in-clinic diagnosis of canine angiostrongylosis. Parasit Vect. (2014) 7:72. doi: 10.1186/1756-3305-7-72

24. Deak G, Gherman CM, Ionică AM, Daskalaki AA, Matei IA, D’Amico G, et al. Use of a commercial serologic test for Angiostrongylus vasorum for the detection of A. chabaudi in wildcats and A. daskalovi in badgers. Vet Parasitol. (2017) 233:107-10. doi: 10.1016/j.vetpar.2016.12.006

25. Liu J, Schnyder M, Willesen JL, Potter A, Chandrashekar R. Performance of the Angio Detect ${ }^{\mathrm{TM}}$ in-clinic test kit for detection of Angiostrongylus vasorum infection in dog samples from Europe. Vet Parasitol Reg Stud Rep. (2017) 7:45-7. doi: 10.1016/j.vprsr.2016.12.007

26. Schnyder M, Tanner I, Webster P, Barutzki D, Deplazes P. An ELISA for sensitive and specific detection of circulating antigen of Angiostrongylus vasorum in serum samples of naturally and experimentally infected dogs. Vet Parasitol. (2011) 179:152-8. doi: 10.1016/j.vetpar.2011.01.054

27. Ferdushy T, Hasan MT. Angiostrongylus vasorum: the 'French Heartworm'. Parasitol Res. (2010) 107:765-71. doi: 10.1007/s00436-010-2026-4 
28. Santoro M, D’Alessio N, Di Prisco F, Neola B, Restucci B, Pagano TB, et al. Angiostrongylus vasorum infection in red foxes (Vulpes vulpes) in southern Italy. Acta Parasitol. (2015) 60:356-69. doi: 10.1515/ap-2015-0050

29. Webster P, Monrad J, Kapel CM, Kristensen AT, Jensen AL, Thamsborg SM. The effect of host age and inoculation dose on infection dynamics of Angiostrongylus vasorum in red foxes (Vulpes vulpes). Parasit Vect. (2017) 10:4. doi: 10.1186/s13071-016-1940-4

30. Woolsey ID, Webster P, Thamsborg S, Schnyder M, Monrad J, Kapel CMO. Repeated inoculations with the lung and heartworm nematode Angiostrongylus vasorum result in increasing larval excretion and worm burden in the red fox (Vulpes vulpes). Int J Parasitol Parasites Wildl. (2017) 6:139-45. doi: 10.1016/j.ijppaw.2017.06.005

31. Di Cesare A, Crisi PE, Di Giulio E, Veronesi F, Frangipane di Regalbono A, Talone T, et al. Larval development of the feline lungworm Aelurostrongylus abstrusus in Helix aspersa. Parasitol Res. (2013) 112:3101-8. doi: 10.1007/s00436-013-3484-2

32. Di Cesare A, Crisi PE, Bartolini R, Iorio R, Talone T, Filippi L, et al. Larval development of Angiostrongylus vasorum in the land snail Helix aspersa. Parasitol Res. (2015) 114:3649-55. doi: 10.1007/s00436-015-4592-y
33. Colella V, Cavalera MA, Deak G, Tarallo VD, Gherman CM, Mihalca AD, et al. Larval development of Angiostrongylus chabaudi, the causative agent of feline angiostrongylosis, in the snail Cornu aspersum. Parasitology. (2017) 144:1922-30 doi: 10.1017/S0031182017001433

Conflict of Interest: NP was employed by the company IDEXX Laboratories, Inc.

The remaining authors declare that the research was conducted in the absence of any commercial or financial relationships that could be construed as a potential conflict of interest.

Copyright (C) 2020 Di Cesare, Morelli, Colombo, Simonato, Veronesi, Marcer, Diakou, D'Angelosante, Pantchev, Psaralexi and Traversa. This is an open-access article distributed under the terms of the Creative Commons Attribution License (CC $B Y)$. The use, distribution or reproduction in other forums is permitted, provided the original author(s) and the copyright owner(s) are credited and that the original publication in this journal is cited, in accordance with accepted academic practice. No use, distribution or reproduction is permitted which does not comply with these terms. 